\title{
Linear Logistic Regression Predicts Generalized Sedation As Well As Response Surface Models For Midazolam And Alfentanil Sedation In Gastrointestinal Endoscopy
}

\author{
Jing-Yang Liou, Shih-Pin Lin, Chien-Kun Ting, Wen-Kuei Chang, Mei-Yung Tsou \\ Department of Anesthesiology, Taipei Veterans General Hospital, Taipei, Taiwan, ROC \\ National Yang-Ming University and School of Medicine, Taipei, Taiwan, ROC
}

Background and Objectives: Researchers have used the linear logistic regression (LR) and the non-linear response surface models (RSMs) to predict patient responses. The reduced Greco and hierarchy RSMs have proven to be accurate in gastrointestinal endoscopies using midazolam and alfentanil. In this study, we evaluate how a simpler model such as LR will perform as compared to the RSMs.
Methods: $\quad$ Patients who received esophagogastroduodenoscopy (EGD) and colonoscopy sedation with midazolam and alfentanil were candidates for the study. LR was performed for the EGD group and validated using the colonoscopy group. The two RSMs went through the same process. Their performance and ROC curves were evaluated.

Results: Thirty-three patients were included and the native EGD LR model had an ROC curve area of 0.94. For external validation, ROC curve was $0.92,0.94$ and 0.94 for the reduced Greco RSM, hierarchy RSM and LR model respectively. Pairwise comparison was not significant.
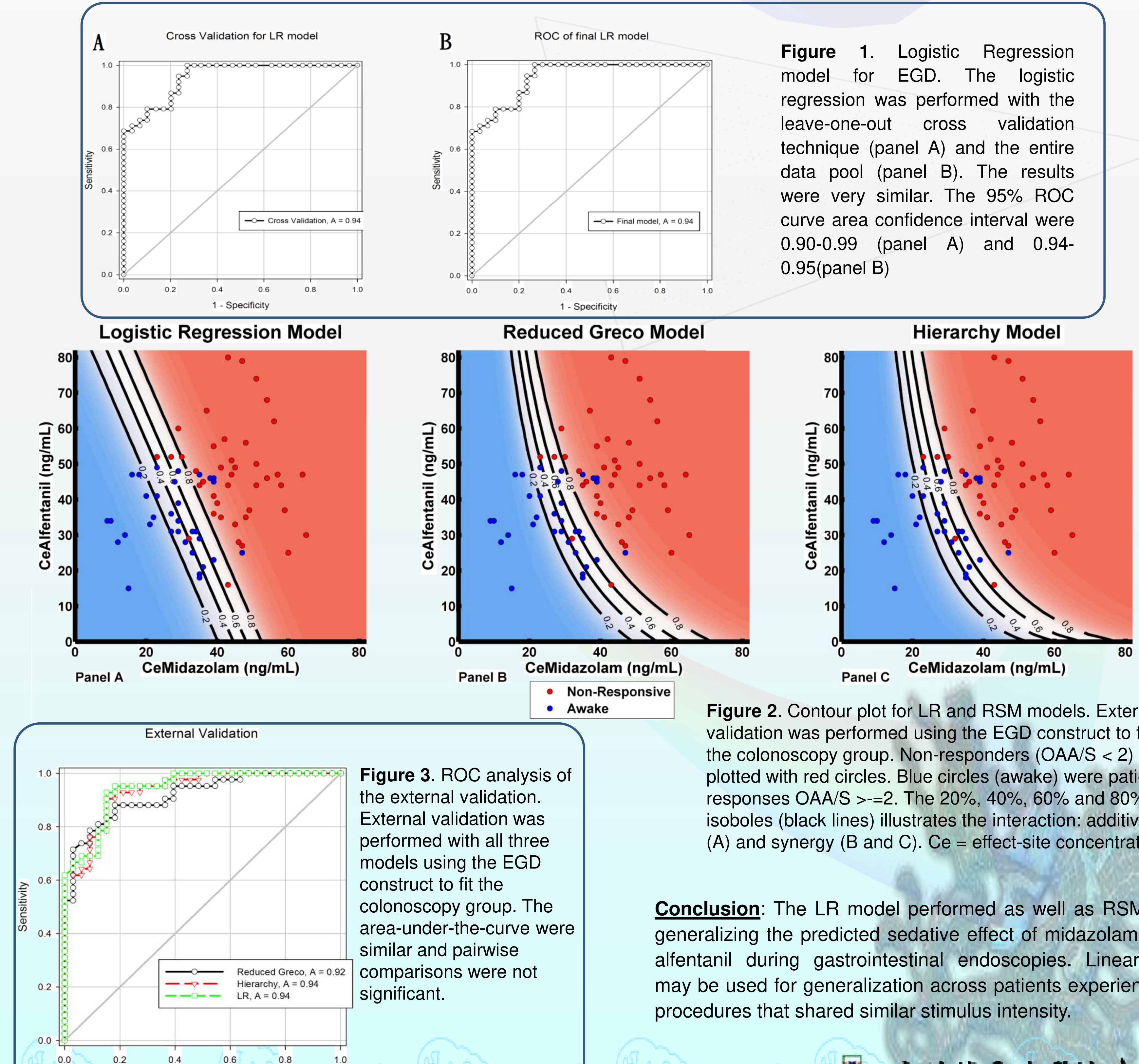

Figure 3. ROC analysis of the external validation. External validation was performed with all three models using the EGD construct to fit the colonoscopy group. The area-under-the-curve were similar and pairwise comparisons were not significant.

Figure 2. Contour plot for LR and RSM models. External validation was performed using the EGD construct to fit the colonoscopy group. Non-responders $(\mathrm{OAA} / \mathrm{S}<2)$ was plotted with red circles. Blue circles (awake) were patient responses OAA/S >- $=2$. The $20 \%, 40 \%, 60 \%$ and $80 \%$ isoboles (black lines) illustrates the interaction: additivity (A) and synergy $(\mathrm{B}$ and $\mathrm{C})$. $\mathrm{Ce}=$ effect-site concentration

Conclusion: The LR model performed as well as RSMs in generalizing the predicted sedative effect of midazolam and alfentanil during gastrointestinal endoscopies. Linear LR may be used for generalization across patients experiencing procedures that shared similar stimulus intensity. 